\title{
Posterior Reversible Encephalopathy Syndrome (PRES) in Haemolytic Anaemia - A Case Report
}

\author{
Aayush Somani ${ }^{1}$, Shilpa Abbay Gaidhane ${ }^{2}$, Priti Abbay Gaidhane ${ }^{3}$, Nazli Khatib ${ }^{4}$, Sourya Acharya ${ }^{5}$ \\ 1,2,3,4,5 Department of Medicine, Jawaharlal Nehru Medical College, Datta Meghe \\ Institute of Medical Sciences (DU), Wardha, Sawangi (M) Maharashtra, India.
}

\section{INTRODUCTION}

Posterior reversible encephalopathy syndrome is a sporadic disease commonly presenting as headache, vomiting, visual disturbances, seizures and confusion. Acute changes in blood pressure are commonly associated with this. ${ }^{1}$ It is understood that many medical conditions and medicines raise the risk of posterior reversible encephalopathy syndrome. We report an unusual case of posterior reversible encephalopathy syndrome in patient with autoimmune haemolytic anaemia.

Due to the advancement and affordability of brain imaging with magnetic resonance imaging (MRI), identification of posterior reversible encephalopathy syndrome (PRES) is possible. A high index of suspicion and prompt treatment can decrease the morbidity, decrease mortality and result in an early recovery.

Haemolysis is the premature destruction of erythrocytes. Haemolysis expedites to haemolytic anaemia when the increased loss of red blood cells cannot be replaced by bone marrow activity. Immune haemolysis is a shortening of the survival of red blood cells due to antibodies, either directly or indirectly. Autoantibodies or alloantibodies can be such antibodies. In order to choose suitable blood for transfusion, these atypical antibodies have to be detected in the patient's serum.

\section{PRESENTATION OF CASE}

We present a case of 23-year-old female, a known case of autoimmune haemolytic anaemia diagnosed 1 month back. She came to our tertiary care hospital in emergency department with history of headache, nausea, vomiting followed by generalised tonic-clonic seizures 3 - 4 episodes prior to admission.

On examination, she was afebrile with pulse rate of 100 beats / min regular, blood pressure was 190 / $110 \mathrm{~mm}$ of Hg in right arm, pallor was present and other general physical examination findings were normal. On admission, she was drowsy, pupils bilaterally equal and reacting to light, no neck stiffness and moving all four limbs. Fundus examination was normal. Cardiovascular, respiratory and abdominal examination was normal.
Corresponding Author: Dr. Shilpa Abbay Gaidhane, Department of Medicine, Jawaharlal Nehru Medical College, Datta Meghe Institute of Medical Sciences (DU), Wardha,

Sawangi, Maharashtra, India.

E-mail: drshilpagaidhane@gmail.com

DOI: $10.14260 / \mathrm{jemds} / 2021 / 140$

How to Cite This Article:

Somani A, Gaidhane SA, Gaidhane PA, et al. Posterior reversible encephalopathy syndrome (PRES) in haemolytic anaemia a case report. J Evolution Med Dent Sci 2021;10(09):656-658, $10.14260 /$ jemds $/ 2021 / 140$

Submission 02-11-2020,

Peer Review 05-01-2021,

Acceptance 12-01-2021,

Published 01-03-2021.

Copyright (c) 2021 Aayush Somani et al. This is an open access article distributed under Creative Commons Attribution License [Attribution 4.0 International (CC BY 4.0)] 


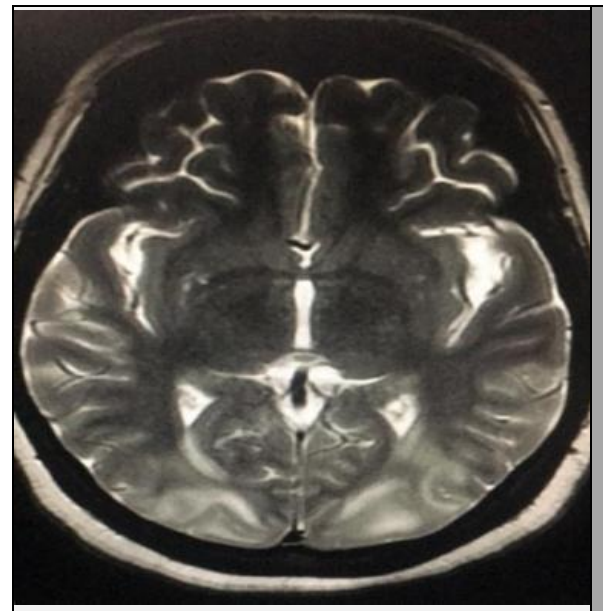

Figure A. T2W Images Showing Hyperintense Lesion in Occipital

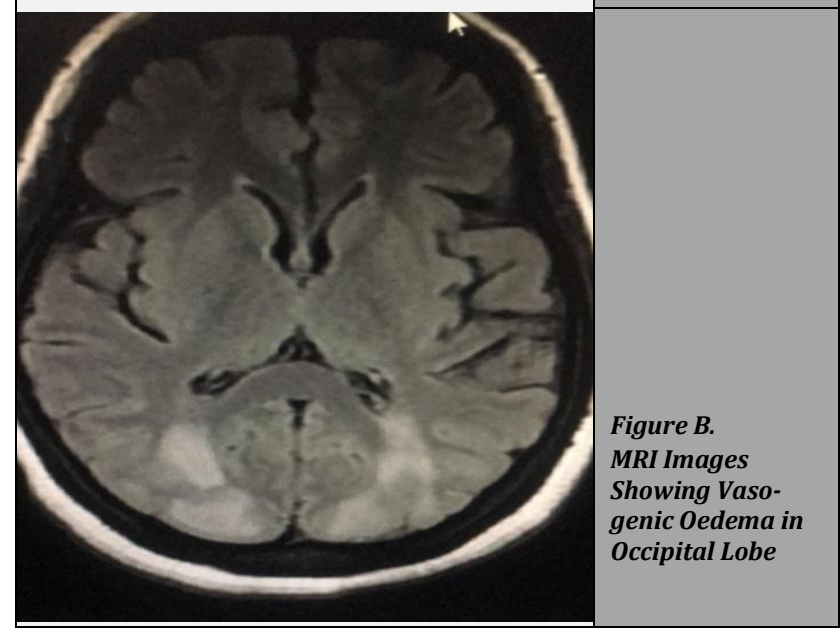

\section{DISCUSSION OF MANAGEMENT}

Patient landed up in status epilepticus. She received $4 \mathrm{mg}$ of intravenous diazepam followed by $1000 \mathrm{mg}$ of intravenous levetiracetam followed by phenytoin $1000 \mathrm{mg}$. As seizures were not controlled, midazolam infusion was started at $15 \mathrm{mg}$ per hour with daily dosing of levetiracetam and phenytoin. After midazolam infusion, her seizures were controlled. In view of high blood pressure, tablet amlodipine $10 \mathrm{mg}$ was given, blood pressure monitoring was done and thereafter there was no requirement of any anti-hypertensive medication. MRI Brain was done suggestive of multiple posterior periventricular and subcortical T2 / FLAIR hyperintensities noted in bilateral occipital, high parietal and right cerebellar region appearing isointense on T1 showing no DWI restriction and blooming on gradient recalled echo (GRE).

Laboratory studies showed haemoglobin $11.4 \mathrm{gm} / \mathrm{dL}$, WBC 4500 cells $/ \mathrm{mm}^{3}$ (neutrophils - $58 \%$ ), platelets 3 lakhs / cumm. Arterial blood gas analysis showed pH-7.32, partial pressure of oxygen (P02)-286, partial pressure of carbon dioxide (PCO2)-38, and bicarbonate concentration (HCO3)-22. Blood sugar was $134 \mathrm{mg} / \mathrm{dL}$. Serum electrolytes, liver function test, kidney function test and routine urine analysis were normal. Cerebrospinal fluid (CSF) showed 2 cells with $100 \%$ lymphocytes with normal protein and sugar. Blood, urine and endotracheal cultures were negative for any growth. Electroencephalogram (EEG) was normal. Chest x-ray, electrocardiogram (ECG) and 2D echocardiogram were also normal.
In view of clinical and radiological findings, posterior reversible encephalopathy syndrome due to autoimmune haemolytic anaemia was diagnosed. She was managed with supportive care- antiepileptics, injection mannitol, antihypertensive, antacid, along with once daily dose of prednisolone $40 \mathrm{mg}$.

\section{DISCUSSION}

Headache, seizures, impaired consciousness and visual hallucinations are symptoms of posterior reversible encephalopathy syndrome (PRES). It is commonly associated with acute increase in blood pressure. It was first described in 1996 by Hinchey et al. ${ }^{2}$ Posterior reversible encephalopathy syndrome has historically been known by different names, such as reversible posterior leukoencephalopathy syndrome, reversible posterior cerebral oedema syndrome and reversible occipital parietal encephalopathy. ${ }^{3}$

There is still no clear exact pathophysiological mechanism of posterior reversible encephalopathy syndrome. Three theories have been suggested so far, including (1) cerebral vasoconstriction that causes subsequent brain infarctions, (2) vasogenic oedema hypothesis is due to failure of the CNS's compensatory mechanism to restrict blood flow in acute hypertension, leading to disturbance of the blood brain barrier that causes brain vasogenic oedema, and (3) acute rise in blood pressure contributes to endothelial damage.

The various aetiology leading to posterior reversible encephalopathy syndrome includes hypertension; preeclampsia; haemolysis, elevated liver enzymes, and low platelet count (HELLP) syndrome; immunosuppressive / cytotoxic drugs; sepsis; systemic lupus erythematosus and nephrotic state. Sepsis and septic shock associated with PRES due to endothelial derangement and microcirculation disturbances. Autoimmune disease like systemic lupus erythematosus, systemic sclerosis, polyarteritis nodosa, Wegener's granulomatosis, thrombotic microangiopathy, polyangiitis, Takayasu-arteritis and Crohn's disease are some of the causes of posterior reversible encephalopathy syndrome. Our patient had autoimmune haemolytic anaemia and the occurrence of posterior reversible encephalopathy syndrome in this disease is rare. There are no specific guidelines for diagnosis of posterior reversible encephalopathy syndrome. The combination of clinical manifestations and radiological criteria establishes the diagnosis of posterior reversible encephalopathy syndrome. ${ }^{4,5}$

Occurrence of haemolysis can be intravascularly, extravascularly in the reticuloendothelial system. Phagocytosis is the main extravascular pathway due to RBC's failure to change form enough to move through the spleen. The intravascular mechanisms include direct cellular destruction, fragmentation and oxidation. ${ }^{6}$ Autoimmune haemolytic anaemia is a moderately rare condition, estimated to be incidence of 1 - 3 cases per 100000 per annum. 48 - 70 percent of warm autoantibodies are responsible for cases of autoimmune haemolytic anaemia. Detection and recognition of RBC alloantibodies is the most pressing issue, which the autoantibodies can mask.

Posterior reversible encephalopathy syndrome (PRES) is reversible neuroradiological clinical entity. The frequency of reversible posterior encephalopathy syndrome is greater in 
women than in men. The mean presentation age is 44 years, with an extended range of between 14 and 78 years. PRES symptoms are usually reversible, although cerebral haemorrhage or ischemia can occur in some situations, leading to permanent neurological deficits or death. ${ }^{7}$ The exact mechanism for posterior reversible encephalopathy syndrome is still unknown. Early diagnosis of PRES is critical to prevent irreversible neurological damage and mortality. PRES can also be a misnomer as the picture changes and clinical characteristics may not be limited to the posterior cerebral hemispheres. The reversibility of PRES may also be clinically or radiologically incomplete; ischemic or haemorrhagic stroke may exacerbate the disease, and may lead to recurrent seizure disorder or death. ${ }^{8}$

Early identification of radiological manifestations is important to the diagnosis. Clinical symptoms are important to the diagnosis, but the clinical symptoms can be non-specific or resemble other neurological diseases. In the adult population, manifestations of PRES include seizures, visual problems and headaches. Usage of intravenous fluids, sedatives, analgesia and supportive treatment and the prevention of possible triggering factors. ${ }^{9}$ are important. Headache is the most prevalent clinical presentation, accompanied by disturbances in the consciousness, which can range from lethargy to coma and somnolence.

Aggressive regulation of blood pressure and management of underlying pathology is the normal treatment for neurological findings. ${ }^{8}$ Especially in some cases of the paediatric population, the blood pressure can only be minimally high or fluctuating during the presentation of PRES. ${ }^{9}$ The initial assessment of PRES patients should concentrate on rapid correction of blood pressure, the use of crystalloid fluids for hydration and maintaining ample oxygenation. PRES syndrome should always be considered. ${ }^{10}$ in women with acute hypertensive disorders associated with epileptic seizures or other neurological symptoms during pregnancy and postpartum. Rapid increase in blood pressure in hypertensives with irregular cerebral auto-regulation contributes to breakage of blood brain barrier and consequent leaking of plasma. ${ }^{11}$ In addition to anti-hypertensive medications, anticonvulsants and supportive treatment showed drastic improvement in PRES.

Usually, PRES is diagnosed on the basis of clinical symptoms, imaging analysis (computed tomography or images of magnetic resonance). ${ }^{7}$ The MRI is the golden standard and only $50 \%$ of the lesions were revealed by the CT scan. ${ }^{8}$ In order to identify symptoms of cytotoxic oedema, which is a symptom of the progression of the disease, PRES clinicians should be aware of cerebral MRI in suspected case. ${ }^{10}$ An early diagnosis is necessary to manage the long- and shortterm complications and morbidity and prevention of death.

A high index of effective therapy will decrease morbidity, decrease mortality and show the path to early recovery. In rapid and large blood transfusions, posterior reversible encephalopathy syndrome may become a major issue.6,7 Early and late complications such as pulmonary oedema, extracranial inner left dissection carotid artery, brain herniation, memory loss in the short term, haemorrhage of sub arachnoids, permanent mild dysmetria, visual dysmetria impairment as well as death has been identified. ${ }^{10}$ PRES management involves the timely delivery of treatment with maintenance of normal blood pressure. When treating patients with altered mental state and seizures, especially in pregnant patients, a high index of suspicion should be maintained. ${ }^{11,12}$

Financial or other competing interests: None.

Disclosure forms provided by the authors are available with the full text of this article at jemds.com.

\section{REFERENCES}

[1] McKinney AM, Short J, Truwit CL, et al. Posterior reversible encephalopathy syndrome: incidence of atypical regions of involvement and imaging findings. AJR Am J Roentgenol 2007;189(4):904-12.

[2] Hinchey J, Chaves C, Appignani B, et al. A reversible posterior leukoencephalopathy syndrome. N Engl J Med 1996;334(8):494-500.

[3] Bartynski WS. Posterior reversible encephalopathy syndrome, part 1: fundamental imaging and clinical features. AJNR Am J Neuroradiol 2008;29(6):1036-42.

[4] Sweany JM, Bartynski WS, Boardman JF. Recurrent posterior reversible encephalopathy syndrome: report of 3 cases--PRES can strike twice! J Comput Assist Tomogr 2007;31(1):148-56.

[5] Sato Y, Ito $\mathrm{T}$, Iguchi $\mathrm{A}$, et al. Posterior reversible encephalopathy syndrome and microangiopathic haemolytic anaemia developing in a regularly haemodialysed patient with scleroderma renal crisis: a case report. Modern Rheumatology Case Reports 2019;3(2):124-9.

[6] Phillips J, Henderson AC. Hemolytic anemia: evaluation and differential diagnosis. Am Fam Physician 2018;98(6):354-61.

[7] $\mathrm{Hu} \mathrm{H}, \mathrm{Xu} \mathrm{S}, \mathrm{Hu} \mathrm{S}$, et al. The clinical characteristics of posterior reversible encephalopathy syndrome in patients with chronic renal failure. Exp Ther Med 2017;14(1):881-7.

[8] Maasoumeh M, Maryam-Sadat H, Maasoumeh S, et al. Posterior reversible encephalopathy syndrome (PRES) associated with eclampsia: a case study. International Journal of Medical Research \& Health Sciences 2017;6(3):48-53.

[9] Gamie Z, Rizwan A, Balen FG, et al. Posterior reversible encephalopathy syndrome in a child with cyclical vomiting and hypertension: a case report. Journal of Medical Case Reports 2011;5(1):137.

[10] Marcoccia E, Piccioni MG, Schiavi MC, et al. Postpartum Posterior Reversible Encephalopathy Syndrome (PRES): three case reports and literature review. Case Rep Obstetr Gynecol 2019;2019:9527632.

[11] Kumar N, Singh R, Sharma N, et al. Atypical presentation of posterior reversible encephalopathy syndrome: two cases. J Anaesthesiol Clin Pharmacol 2018;34(1):120-2.

[12] Kaur P, Basu S, Kaur R, et al. Immune hemolytic anemia: a report of two cases. J Lab Physicians 2009;1(1):22-4. 